\title{
Impact of a systolic parameter, defined as the ratio of right brachial pre-ejection period to ejection time, on the relationship between brachial-ankle pulse wave velocity and left ventricular diastolic function
}

\author{
Po-Chao Hsu ${ }^{1}$, Tsung-Hsien Lin ${ }^{1,2}$, Chee-Siong Lee ${ }^{1,2}$, Chun-Yuan Chu ${ }^{1}$, Ho-Ming Su ${ }^{1,2,3}$, Wen-Chol Voon ${ }^{1,2}$, \\ Wen-Ter Lai ${ }^{1,2}$ and Sheng-Hsiung Sheu ${ }^{1,2}$
}

Arterial stiffness is correlated with left ventricular (LV) diastolic function as well as susceptibility to LV systolic function. Therefore, if LV systolic function is not known, the relationship between arterial stiffness and LV diastolic function is difficult to determine. A total of 260 patients were included in the study. The brachial-ankle pulse wave velocity (baPWV) and the ratio of right brachial pre-ejection period to ejection time (rbPEP/rbET) were measured using an ABI-form device. Patients were classified into four groups. Groups 1, 2, 3 and 4 were patients with rbPEP/rbET and baPWV below the median, rbPEP/rbET above but baPWV below the median, rbPET/rbET below but baPWV above the median, and rbPET/rbET and baPWV above the median, respectively. The LV ejection fractions in groups 1 and 3 were higher than those in groups 2 and $4(P<0.001$ for all). Patients in group 1 had a lower left atrial volume index (LAVI) and higher early diastolic mitral annular velocity (Ea) than patients in the other groups $(P \leq 0.002)$. Patients in group 2 had a LAVI and ratio of transmitral $E$ wave velocity to Ea that were comparable to those in groups 3 and 4 . In conclusion, rbPEP/rbET had an impact on the relationship between baPWV and LV diastolic function. In patients with high rbPEP/rbET but low baPWV, low baPWV may not indicate good LV diastolic function but implies that cardiac dysfunction may precede vascular dysfunction in such patients. When interpreting the relationship between baPWV and LV diastolic function, the rbPEP/rbET value obtained from the same examination should be considered.

Hypertension Research (2011) 34, 462-467; doi:10.1038/hr.2010.262; published online 13 January 2011

Keywords: arterial stiffness; ejection time; left ventricular diastolic function; pre-ejection period; pulse wave velocity

\section{INTRODUCTION}

Increased pulse wave velocity (PWV), which reflects increased arterial stiffness, is reported to be an independent predictor of cardiovascular outcomes $^{1,2}$ and is associated with the presence of coronary artery narrowing. ${ }^{3}$ Masugata et al. ${ }^{4}$ found that brachial-ankle PWV (baPWV) was significantly correlated with left ventricular (LV) diastolic function. Abhayaratna et al. ${ }^{5}$ demonstrated that aortic PWV progressively increased according to the severity of diastolic dysfunction and had a significantly negative correlation with early diastolic mitral annular velocity (Ea). Several studies have also reported a relationship between arterial stiffness and LV diastolic function. ${ }^{6-8}$ In these studies, ${ }^{4-8}$ they chiefly included patients with preserved LV systolic function. However, one of our recent studies revealed that there was a mismatch between increases in arterial stiffness and LV diastolic dysfunction in patients with wide range of LV systolic function. ${ }^{9}$ Weber et al. also evaluated the relationship between aortic PWV and LV systolic function in 63 patients with cardiomyopathy (LV ejection fraction <45\%) and 126 controls (LV ejection fraction $>50 \%$ ), and found that aortic PWV did not differ between cardiomyopathy patients and controls, and that aortic PWV had a significant positive correlation with LV ejection fraction in cardiomyopathy patients. Thus, they concluded that aortic PWV values were susceptible to LV performance. ${ }^{10}$ Therefore, if LV systolic function is not known, the correlation between PWV and LV diastolic function is difficult to determine.

A clinical device, ABI-form (Colin VP1000, Komaki, Japan), has been developed to automatically and simultaneously measure blood pressure (BP) in both arms and ankles, and record pulse waves of the

${ }^{1}$ Division of Cardiology, Department of Internal Medicine, Kaohsiung Medical University Hospital, Kaohsiung Medical University, Kaohsiung, Taiwan, ROC; ${ }^{2}$ Department of Internal Medicine, Faculty of Medicine, College of Medicine, Kaohsiung Medical University, Kaohsiung, Taiwan, ROC and ${ }^{3}$ Department of Internal Medicine, Kaohsiung Municipal Hsiao-Kang Hospital, Kaohsiung, Taiwan, ROC

Correspondence: Dr H-M Su, Department of Internal Medicine, Kaohsiung Municipal Hsiao-Kang Hospital, Kaohsiung Medical University, 482 Shan-Ming Road, Hsiao-Kang District, 812 Kaohsiung, Taiwan, ROC.

E-mail: cobeshm@seed.net.tw

Received 16 May 2010; revised 6 October 2010; accepted 7 October 2010; published online 13 January 2011 
brachial and posterior tibial arteries by an automated oscillometric method. Using this device, we can easily obtain baPWV values. ${ }^{11}$ In addition, this device can automatically calculate the right brachial preejection period (rbPEP) and right brachial ejection time (rbET) by analyzing the signals of electrocardiograms, phonocardiograms and right brachial pressure volume waveforms. Therefore, we can obtain baPWV and rbPEP/rbET values during the same examination. Recently, we have shown that rbPEP/rbET has a significant correlation with LV ejection fraction and is a useful parameter in predicting impaired LV systolic function. ${ }^{12}$ As depressed LV systolic function may affect baPWV, rbPEP/rbET may influence the relationship between baPWV and LV diastolic function. The aim of this study was to compare LV diastolic function among patients with $\mathrm{rbPEP} / \mathrm{rbET}$ and baPWV below the median, rbPEP/rbET above but baPWV below the median, rbPEP/rbET below but baPWV above the median, and $\mathrm{rbPEP} / \mathrm{rbET}$ and baPWV above the median and to assess the impact of $\mathrm{rbPEP} / \mathrm{rbET}$ on the relationship between baPWV and LV diastolic function.

\section{METHODS}

\section{Study subjects}

Study subjects were randomly included from a group of patients who arranged for echocardiographic examinations at Kaohsiung Municipal Hsiao-Kang Hospital. Patients with significant aortic or mitral valve disease, atrial fibrillation or inadequate image visualization were excluded. We did not include all patients consecutively because baPWV and rbPEP/rbET must be measured within $5 \mathrm{~min}$ after the completion of an echocardiographic examination. In total, 260 patients were randomly included in this study. The study population was further classified into four groups on the basis of $\mathrm{rbPEP} / \mathrm{rbET}$ and baPWV measurements. Groups 1,2, 3 and 4 were made up of patients with rbPEP/rbET and baPWV both below the median, rbPEP/rbET above but baPWV below the median, rbPET/rbET below but baPWV above the median, and rbPET/rbET and baPWV both above the median, respectively. All patients were in sinus rhythm. The study protocol was approved by our Institutional Review Board and all enrolled patients gave written informed consent.

\section{Echocardiographic assessment}

The echocardiographic examinations were performed by one experienced sonographer using transthoracic echocardiography (Vivid 7, General Electric Medical Systems, Horten, Norway), with the participant respirating quietly in the left decubitus position. Two-dimensional and two-dimensionally guided $\mathrm{M}$-mode images were recorded from the standardized views. The Doppler sample volume was placed at the tips of the mitral leaflets to obtain the LV inflow waveforms from the apical 4-chamber view. All sample volumes were positioned with ultrasonic beam alignment to flow. Tissue Doppler imaging was obtained with the sample volume placed at the lateral corner of the mitral annulus from the apical 4-chamber view. The wall filter settings were adjusted to exclude high-frequency signals, and the gain was minimized. A normal mitral inflow pattern was recognized if the ratio of transmitral $\mathrm{E}$ wave velocity (E) to transmitral A wave velocity (A) was $\geq 0.75, \mathrm{Ea} \geq 8 \mathrm{~cm} \mathrm{~s}^{-1}$, and $\mathrm{E} / \mathrm{Ea} \leq 10$, impaired relaxation mitral inflow pattern if the $\mathrm{E} / \mathrm{A}$ ratio was $<0.75$, and pseudonormal/restrictive mitral inflow pattern if the E/A ratio was $\geq 0.75$, $\mathrm{Ea}<8 \mathrm{~cm} \mathrm{~s}^{-1}$ or $\mathrm{E} / \mathrm{Ea}>10 .{ }^{13,14}$ The LV ejection fraction was measured by the modified Simpson's method. The left atrial volume was measured by the biplane area-length method. ${ }^{15}$ Apical 4- and 2-chamber views were obtained to determine the left atrial area and length (from the middle of the plane of the mitral annulus to the posterior wall). The maximal left atrial chamber area and length were measured before mitral valve opening, excluding the left atrial appendage and pulmonary veins. The left atrial volume was calculated and indexed to the body surface area. The raw ultrasound data were recorded and analyzed offline, using software (EchoPAC, GE Medical Systems), by a cardiologist who was blinded to the other data.

\section{Assessment of baPWV, rbPEP and rbET}

Within $5 \mathrm{~min}$ after the completion of the echocardiographic examination baPWV, rbPEP and rbET were assessed using an ABI-form device (VP1000, Colin, Aichi, Japan), which automatically and simultaneously measures BP in both arms and ankles by an oscillometric method. ${ }^{16,17}$ For measuring baPWV, pulse waves that were obtained from the brachial and tibial arteries were recorded simultaneously, and the transmission time, which was defined as the time interval between the initial increase in brachial and tibial waveforms, was determined. The transmission distance from the arm to each ankle was calculated according to body height. The value of baPWV was automatically computed as the transmission distance divided by the transmission time. After obtaining bilateral baPWV values, the average of two values was used for analysis. Systolic and diastolic BPs were measured by the same device. The average of systolic and diastolic BP of bilateral arms was used for analysis. The rbET was automatically measured from the foot to the dicrotic notch (equivalent to the incisura on the down-stroke of the aortic pressure wave contour produced by the closure of the aortic valve) of the pulse volume waveform. The total electromechanical systolic interval $\left(\mathrm{QS}_{2}\right)$ was measured from the onset of the QRS complex on the electrocardiogram to the first highfrequency vibrations of the aortic component of the second heart sound on the phonocardiogram. The rbPEP was automatically calculated by subtracting rbET from $\mathrm{QS}_{2} \cdot{ }^{12,18}$

\section{Statistical analysis}

All data were expressed as mean values ( \pm s.d.). SPSS 11.0 software (SPSS, Chicago, IL, USA) was used for statistical analysis. Multiple comparisons between study groups were performed by one-way analysis of variance followed by a post hoc test adjusted with a Bonferroni correction. Categorical and continuous variables between groups were compared by $\chi^{2}$ analysis and an independent-samples $t$-test, respectively. The relationship between two continuous variables was assessed by a bivariate correlation method (Pearson's correlation). All tests were 2-sided, and the level of significance was established as $P<0.05$.

\section{RESULTS}

The median values of baPWV and $\mathrm{rbPEP} / \mathrm{rbET}$ were $1623 \mathrm{~cm} \mathrm{~s}^{-1}$ and 0.352 , respectively. The comparison of clinical characteristics among the study groups is shown in Table 1. There were 68, 62, 62 and 68 patients in groups $1,2,3$ and 4 , respectively. There were significant differences among the four groups in age, prevalence of diabetes mellitus and hypertension, heart rate, systolic and diastolic BP and pulse pressure. The study was performed with patients who were taking their usual medications.

The comparisons of echocardiographic characteristics, baPWV and $\mathrm{rbPEP} / \mathrm{rbET}$ among the study groups are shown in Table 2 . There were significant differences among the four groups in the left atrial volume index (LAVI), LV end-diastolic dimension, LV end-systolic dimension, LV ejection fraction, E, A, E/A, E-wave deceleration time, Ea, E/Ea, late diastolic velocity of lateral mitral annulus, rbPEP/rbET, baPWV and the percentage of mitral inflow patterns. Furthermore, LAVI was smaller in group 1 than in the other groups $(P \leq 0.002)$, but did not differ between groups 2 and $3(P=1.00)$ or between groups 2 and 4 $(P=1.00)$. The $\mathrm{LV}$ ejection fraction was higher in groups 1 and 3 than in groups 2 and $4(P<0.001$ for all). Ea was higher in group 1 than in the other groups $(P<0.001$ for all $)$ and was higher in group 2 than in groups 3 and $4(P \leq 0.011)$. E/Ea was lower in group 1 than in groups 3 and $4(P \leq 0.001)$, but did not differ between groups 2 and 3 $(P=1.00)$ or between groups 2 and $4(P=0.28)$.

The univariate correlates of baPWV and $\mathrm{rbPEP} / \mathrm{rbET}$ are shown in Table 3. Although baPWV and rbPEP/rbET had significant correlations with many parameters of LV diastolic and systolic function, $\mathrm{rbPEP} / \mathrm{rbET}$ had no correlation with baPWV $(r=0.015, P=0.813)$. Age had a positive correlation with baPWV $(r=0.625, P<0.001), \mathrm{E} / \mathrm{Ea}$ 
Table 1 Comparison of clinical characteristics among study groups

\begin{tabular}{|c|c|c|c|c|c|}
\hline & Group1 & Group 2 & Group 3 & Group 4 & P \\
\hline Age (years) & $50 \pm 11$ & $49 \pm 13$ & $68 \pm 10^{* \dagger}$ & $61 \pm 12^{* \dagger \#}$ & $<0.001$ \\
\hline $\mathrm{DM}(\%)$ & $6(8.8 \%)$ & $16(25.8 \%)^{*}$ & $21(33.9 \%)^{*}$ & $26(38.2 \%)^{*}$ & 0.001 \\
\hline $\mathrm{H} / \mathrm{T}(\%)$ & $24(35.3 \%)$ & $32(51.6 \%)$ & $48(77.4 \%)^{\star \dagger}$ & $52(76.5 \%)^{* \dagger}$ & $<0.001$ \\
\hline BMI $\left(\mathrm{kg} \mathrm{m}^{-2}\right)$ & $24.8 \pm 3.4$ & $26.2 \pm 4.4$ & $25.1 \pm 3.5$ & $25.1 \pm 3.6$ & 0.15 \\
\hline$\beta$ blockers (\%) & 24 (35.3\%) & 33 (53.2\%) & 22 (35.5\%) & 31 (45.6\%) & 0.118 \\
\hline CCBs $(\%)$ & $9(13.2 \%)$ & $8(12.9 \%)$ & $24(38.7 \%)^{* \dagger}$ & $23(33.8 \%)^{* \dagger}$ & $<0.001$ \\
\hline ACEIs (\%) & $7(10.3 \%)$ & $16(25.8 \%)^{*}$ & $7(11.3 \%)$ & $12(17.6 \%)^{\dagger}$ & 0.066 \\
\hline ARBs $(\%)$ & $12(17.6 \%)$ & $15(24.2 \%)$ & $23(37.1 \%)^{*}$ & $31(45.6 \%)^{*}$ & 0.002 \\
\hline Heart rate (beats per min) & $66 \pm 8$ & $73 \pm 14^{*}$ & $68 \pm 9$ & $77 \pm 16^{* \#}$ & $<0.001$ \\
\hline $\mathrm{SBP}(\mathrm{mm} \mathrm{Hg})$ & $127 \pm 15$ & $127 \pm 18$ & $153 \pm 19^{* \dagger}$ & $146 \pm 23^{* \dagger}$ & $<0.001$ \\
\hline
\end{tabular}

Abbreviations: ACEIs, angiotensin-converting enzyme inhibitors; ARBs, angiotensin II receptor antagonists; BMI, body mass index; CCBs, calcium channel blockers; DBP, diastolic blood pressure; $\mathrm{DM}$, diabetes mellitus; $\mathrm{H} / \mathrm{T}$, hypertension; PP, pulse pressure; SBP, systolic blood pressure.

${ }^{*} P<0.05$ compared with group $1 ;{ }^{\dagger} P<0.05$ compared with group 2 ; ${ }^{\#} P<0.05$ compared with group 3.

Table 2 Comparison of echocardiographic characteristics, baPWV and rbPEP/rbET among study groups

\begin{tabular}{|c|c|c|c|c|c|}
\hline & Group 1 & Group 2 & Group 3 & Group 4 & $P$ \\
\hline LAVI $\left(\mathrm{ml} \mathrm{m}^{-2}\right)$ & $28 \pm 10$ & $37 \pm 17^{*}$ & $37 \pm 12^{*}$ & $41 \pm 16^{*}$ & $<0.001$ \\
\hline LVEDD (mm) & $51 \pm 7$ & $55 \pm 10^{*}$ & $52 \pm 8$ & $55 \pm 9^{*}$ & 0.002 \\
\hline LVESD (mm) & $31 \pm 8$ & $40 \pm 13^{*}$ & $33 \pm 9^{\dagger}$ & $39 \pm 13^{* \#}$ & $<0.001$ \\
\hline $\mathrm{EF}(\%)$ & $65 \pm 11$ & $50 \pm 19^{*}$ & $61 \pm 14^{\dagger}$ & $50 \pm 18^{* \#}$ & $<0.001$ \\
\hline LVMI $\left(\mathrm{g} \mathrm{m}^{-2}\right)$ & $92 \pm 33$ & $111 \pm 39^{*}$ & $114 \pm 37^{*}$ & $121 \pm 35^{*}$ & $<0.001$ \\
\hline$E\left(\mathrm{cms}^{-1}\right)$ & $84 \pm 20$ & $81 \pm 18$ & $78 \pm 24$ & $73 \pm 23^{*}$ & 0.019 \\
\hline$A\left(\mathrm{~cm} \mathrm{~s}^{-1}\right)$ & $73 \pm 18$ & $67 \pm 22$ & $91 \pm 18^{* \dagger}$ & $78 \pm 20^{\dagger \#}$ & $<0.001$ \\
\hline$E / A$ & $1.21 \pm 0.37$ & $1.46 \pm 0.92$ & $0.92 \pm 0.50^{\dagger}$ & $1.04 \pm 0.63^{\dagger}$ & $<0.001$ \\
\hline EDT (ms) & $178 \pm 42$ & $169 \pm 59$ & $210 \pm 57^{* \dagger}$ & $185 \pm 59$ & 0.001 \\
\hline $\mathrm{Ea}\left(\mathrm{cm} \mathrm{s}^{-1}\right)$ & $12.1 \pm 3.8$ & $9.6 \pm 3.8^{*}$ & $7.8 \pm 2.9^{* \dagger}$ & $6.9 \pm 2.5^{\star \dagger}$ & $<0.001$ \\
\hline $\mathrm{E} / \mathrm{Ea}$ & $7.7 \pm 3.8$ & $10.2 \pm 6.0$ & $11.6 \pm 5.7^{*}$ & $12.2 \pm 6.5^{*}$ & $<0.001$ \\
\hline $\mathrm{Aa}\left(\mathrm{cm} \mathrm{s}^{-1}\right)$ & $9.6 \pm 2.3$ & $8.2 \pm 3.3^{*}$ & $10.1 \pm 2.6^{\dagger}$ & $9.5 \pm 3.6$ & 0.005 \\
\hline $\mathrm{rbPEP} / \mathrm{rbET}$ & $0.309 \pm 0.036$ & $0.466 \pm 0.105^{*}$ & $0.302 \pm 0.032^{\dagger}$ & $0.438 \pm 0.066^{*} \#$ & $<0.001$ \\
\hline baPWV $\left(\mathrm{cm} \mathrm{s}^{-1}\right)$ & $1385 \pm 137$ & $1413 \pm 127$ & $2019 \pm 488^{* \dagger}$ & $2008 \pm 327^{* \dagger}$ & $<0.001$ \\
\hline Normal mitral inflow pattern & 49 (72\%) & $26(42 \%)^{*}$ & $18(29 \%)^{*}$ & $11(16 \%)^{*} \dot{\dagger}$ & $<0.001$ \\
\hline Impaired relaxation mitral inflow pattern & $8(12 \%)$ & $7(11 \%)$ & $29(47 \%)^{*} \dagger$ & $26(38 \%)^{*} \dagger$ & $<0.001$ \\
\hline Pseudonormal/restrictive mitral inflow pattern & $11(16 \%)$ & $29(47 \%)^{*}$ & $15(24 \%)^{\dagger}$ & $31(46 \%)^{*} \#$ & $<0.001$ \\
\hline
\end{tabular}

$(r=0.241, P<0.001)$ and LAVI $(r=0.167, P=0.008)$ and had a negative correlation with $\mathrm{Ea}(r=-0.512, P<0.001)$. Systolic BP had a positive correlation with baPWV $(r=0.614, P<0.001)$, E/Ea $\quad(r=0.131$, $P=0.036)$, and LAVI $(r=0.153, P=0.015)$ and a negative correlation with Ea $(r=-0.304, P<0.001)$. LV end-diastolic dimension had a positive correlation with E/Ea $(r=0.487, P<0.001)$ and LAVI $(r=0.583, P<0.001)$ and a negative correlation with Ea $(r=-0.352$, $P<0.001)$. LV end-systolic dimension had a positive correlation with E/Ea $(r=0.538, P<0.001)$ and LAVI $(r=0.618, P<0.001)$ and a negative correlation with Ea $(r=-0.373, P<0.001)$.

\section{DISCUSSION}

The main objective of our study was to compare baPWV and LV diastolic function between four groups divided by baPWV and rbPEP/
rbET values, especially between groups 2 and 3. Before comparing cardiovascular function, it is important to clarify the characteristics of these four groups. Compared with patients in group 3, patients in group 2 were younger and composed of fewer patients of hypertension, but cardiac function, especially systolic function, was more strongly decreased. The characteristics of these four groups could generally be defined as follows: patients in group 1 had relative normal cardiovascular function, patients in group 2 had systolic dysfunction, patients in group 3 had vascular dysfunction, and patients in group 4 had cardiovascular dysfunction. Cardiovascular dysfunction progresses with arterial-cardiac interactions, but the progression of dysfunction differs in speed between the heart and the vessels. In some patients, cardiac dysfunction progresses first (group 2), and in other patients, vascular dysfunction progresses predominantly 
Table 3 Univariate correlates of baPWV and rbPEP/rbET

\begin{tabular}{|c|c|c|c|c|}
\hline & \multicolumn{2}{|c|}{ baPWV } & \multicolumn{2}{|c|}{$r b P E P / r b E T$} \\
\hline & r & $P$ & r & $P$ \\
\hline Age (years) & 0.625 & $<0.001$ & -0.306 & $<0.001$ \\
\hline $\mathrm{BMI}\left(\mathrm{kg} \mathrm{m}^{-2}\right)$ & -0.018 & 0.779 & 0.27 & $<0.001$ \\
\hline Heart rate (beats per min) & 0.089 & 0.153 & 0.497 & $<0.001$ \\
\hline SBP $(m m ~ H g)$ & 0.614 & $<0.001$ & -0.077 & 0.217 \\
\hline $\mathrm{DBP}(\mathrm{mm} \mathrm{Hg})$ & 0.404 & $<0.001$ & 0.148 & 0.018 \\
\hline $\mathrm{PP}(\mathrm{mm} \mathrm{Hg})$ & 0.585 & $<0.001$ & -0.244 & $<0.001$ \\
\hline LAVI $\left(\mathrm{ml} \mathrm{m}^{-2}\right)$ & 0.278 & $<0.001$ & 0.369 & $<0.001$ \\
\hline $\operatorname{LVEDD}(\mathrm{mm})$ & 0.111 & 0.075 & 0.392 & $<0.001$ \\
\hline LVESD (mm) & 0.08 & 0.2 & 0.528 & $<0.001$ \\
\hline EF (\%) & -0.126 & 0.042 & -0.539 & $<0.001$ \\
\hline LVMI $\left(\mathrm{g} \mathrm{m}^{-2}\right)$ & 0.351 & $<0.001$ & 0.291 & $<0.001$ \\
\hline $\mathrm{E}\left(\mathrm{cm} \mathrm{s}^{-1}\right)$ & -0.22 & $<0.001$ & 0.019 & 0.763 \\
\hline$A\left(\mathrm{~cm} \mathrm{~s}^{-1}\right)$ & 0.417 & $<0.001$ & -0.347 & $<0.001$ \\
\hline$E / A$ & -0.444 & $<0.001$ & 0.344 & $<0.001$ \\
\hline EDT (ms) & 0.207 & 0.001 & -0.287 & $<0.001$ \\
\hline $\mathrm{Ea}\left(\mathrm{cm} \mathrm{s}^{-1}\right)$ & -0.505 & $<0.001$ & -0.224 & $<0.001$ \\
\hline $\mathrm{E} / \mathrm{Ea}$ & 0.375 & $<0.001$ & 0.197 & 0.001 \\
\hline $\mathrm{Aa}\left(\mathrm{cm} \mathrm{s}^{-1}\right)$ & 0.173 & 0.005 & -0.274 & $<0.001$ \\
\hline rbPEP/rbET & 0.015 & 0.813 & & \\
\hline
\end{tabular}

Abbreviations: A, peak late transmitral filling wave velocity; $A a$, late diastolic velocity of lateral mitral annulus; baPWV, brachial-ankle pulse wave velocity; BMI, body mass index; E, peak early transmitral filling wave velocity; Ea, early diastolic velocity of lateral mitral annulus; EDT, E-wave deceleration time; DBP, diastolic blood pressure; LAVI, left atrial volume index; LVESD, left ventricular end-systolic dimension; LVMI, left ventricular mass index; $r$. Pearson's correlation coefficient; rbET, right brachial ejection time; rbPEP, right brachial pre-ejection period; SBP, systolic blood pressure.

(group 3), but finally, these patients arrive at cardiovascular dysfunction (group 4). Although baPWV and rbPEP/rbET have significant correlations with many parameters of LV diastolic and systolic function, there is no correlation between them, which suggests that these two parameters may be complementary. Hence, dividing study subjects into four groups using baPWV and rbPEP/rbET was useful in staging the progression of cardiovascular dysfunction.

Doppler echocardiography is the non-invasive method of choice for the assessment of diastolic dysfunction. Because of opposing effects of impaired relaxation and filling pressures in the transmitral flow pattern, the conventional Doppler parameters of LV filling, such as E, A, E/A and E-wave deceleration time, displayed bimodal distributions with increasing LV diastolic dysfunction severity. By contrast, Ea, $\mathrm{E} / \mathrm{Ea}$ and LAVI presented unidirectional changes with worsening LV diastolic function. ${ }^{14,19-24}$ This pseudonormal phenomenon as LV diastolic dysfunction increases in E/A but not in Ea, E/Ea and LAVI may explain the different results between E/A and Ea, E/Ea and LAVI in the present study. In addition, previous studies found that decreased $\mathrm{Ea}$ and increased $\mathrm{E} / \mathrm{Ea}$ and LAVI were correlated with LV diastolic dysfunction. ${ }^{19-23}$ The present study demonstrated that $\mathrm{rbPEP} / \mathrm{rbET}$ had an important impact on the relationship between baPWV and LV diastolic function, as indicated by Ea, LAVI and E/Ea values. Patients in group 1 and group 4 had relatively good and poor LV diastolic function, respectively. However, patients in group 2 did not reveal better LV diastolic function, as indicated by LAVI and E/Ea, than patients in group 3. When interpreting the relationship between baPWV and LV diastolic function, rbPEP/rbET should be taken into consideration.

The PWV can be measured at different locations and by different methods. We can detect arterial pulse waves using Doppler ultrasound, ${ }^{25}$ applanation tonometry ${ }^{26}$ and pressure-sensitive transducers. ${ }^{27}$ PWV can also be obtained by magnetic resonance imaging, ${ }^{28}$ but its limitations are its high cost and lack of availability. Increased PWV is an independent predictor of cardiovascular morbidity and mortality in the general population and in hypertensive patients, the elderly, and patients with end-stage renal disease. ${ }^{2,27,29}$ Despite the fact that there are several parameters used in the assessment of arterial stiffness, the gold standard of non-invasive arterial stiffness measurement is carotid-femoral $\mathrm{PWV}^{27}$ In this study, we used baPWV as the parameter of arterial stiffness. Tanaka et al. ${ }^{30}$ demonstrated that baPWV and carotid-femoral PWV were indices of arterial stiffness that exhibit similar extents of associations with cardiovascular disease risk factors and clinical events.

In our study, although there were several factors correlated with baPWV and LV diastolic parameters, the intensity of influence of these factors on baPWV and LV diastolic parameters varied. Previous studies have shown that both baPWV and LV diastolic function are significantly correlated with age. ${ }^{31,32}$ Our results showed that age was strongly correlated with baPWV $(r=0.625, P<0.001)$. Although age had also a strong correlation with $\mathrm{Ea}(r=-0.512, P<0.001)$, it had only weak correlations with E/Ea $(r=0.241, P<0.001)$ and LAVI $(r=0.167, P=0.008)$. Therefore, the impact of age on baPWV may be greater than its impact on LV diastolic parameters. Similarly, systolic BP had a strong correlation with baPWV $(r=0.614$, $P<0.001)$, but only a moderate correlation with Ea $(r=-0.304$, $P<0.001)$ and weak correlations with $\mathrm{E} / \mathrm{Ea}(r=0.131, P=0.036)$ and LAVI $(r=0.153, P=0.015)$. Thus, systolic BP had a large effect on baPWV but only a mild effect on LV diastolic parameters. Although LV end-diastolic dimension and LV end-systolic dimension had no correlation with baPWV, they had a moderate to strong correlation with LV diastolic parameters, as indicated by LAVI, Ea and E/Ea ( $r=-0.373$ to $0.618, P<0.001$ for all). Thus, LV chamber size had a significant correlation with $\mathrm{LV}$ diastolic parameters but no correlation with baPWV. In addition, although $\mathrm{rbPEP} / \mathrm{rbET}$ had no correlation with baPWV, it had a moderate correlation with LV diastolic parameters, including Ea, E/Ea and LAVI $(r=-0.224$ to $0.369, P \leq 0.001$ for all). Therefore, when evaluating the relationship between arterial stiffness and LV diastolic function, these factors should be considered.

Increased age, systolic BP and baPWV and decreased LV systolic function were reported to be associated with decreased LV diastolic function. ${ }^{4-9,33-35}$ The major difference between groups 1 and 2 and between groups 3 and 4 was LV systolic function, and this discrepancy may explain the worse LV diastolic function, as indicated by Ea and LAVI, in group 2 than in group 1 and the higher percentage of pseudonormal/restrictive mitral inflow pattern in group 4 than in group 3. However, LV systolic function was comparable between groups 1 and 3 and between groups 2 and 4 . Hence, compared with patients in group 3, patients in group 1 with baPWV below the median had better LV diastolic function, as indicated by Ea, E/Ea and LAVI. When compared with patients in group 4, patients in group 2 with baPWV below the median had higher Ea but comparable to E/Ea and LAVI. As increased age was reported to be correlated with decreased $\mathrm{Ea},{ }^{33-35}$ the higher Ea in group 2 compared with group 4 may be due to younger average age rather than better LV diastolic function. Hence, in patients with $\mathrm{rbPEP} / \mathrm{rbET}$ above the median, decreased baPWV may not indicate good LV diastolic function. The interpretation of LV diastolic function by way of baPWV must be considered cautiously in these patients. In addition, compared with patients in group 3, patients in group 2 had a younger average age, lower systolic BP and lower baPWV, so they should have better LV diastolic function. In fact, although Ea was higher in group 2 than in group 3, E/Ea and LAVI were comparable between them. Similarly, 
younger age, not better LV diastolic function, in group 2 may explain this higher Ea. Patients in group 2 also had fewer impaired relaxation mitral inflow patterns, but more pseudonormal/restrictive mitral inflow patterns than those in group 3. This finding implied that the LV diastolic dysfunction grade might be more advanced in group 2 than in group 3. As LV diastolic dysfunction generally occurred earlier than systolic dysfunction, the more depressed LV systolic function in group 2 compared with group 3 might explain why LV diastolic function, as indicated by E/Ea and LAVI, in group 2 was comparable with that in group 3 and why the LV diastolic dysfunction grade in group 2 was even more advanced than that of group 3. As Ea had a strong correlation with baPWV $(r=-0.505, P<0.001)$, vascular function should have a large impact on $\mathrm{Ea}$, which might explain why Ea was lower in group 3, which was characterized mainly by vascular dysfunction, than in group 2, which was characterized mainly by systolic dysfunction. In contrast, E/Ea $(r=0.375, P<0.001)$ and LAVI $(r=0.278, P<0.001)$ correlated only moderately with baPWV, but they correlated significantly with $\mathrm{rbPEP} / \mathrm{rbET}$. Hence, vascular and cardiac function might have a similar influence on E/Ea and LAVI, which might explain why E/Ea and LAVI were comparable between groups 2 and 3 . In addition, compared with patients in group 3, patients in group 2 had more strongly depressed LV systolic function. Hence, cardiac dysfunction should precede vascular dysfunction in group 2, which might explain the low baPWV in group 2 .

\section{Study limitations}

The majority of our patients were treated chronically with antihypertensive medications. For ethical reasons, we did not withdraw these medications. Hence, we could not exclude the influence of antihypertensive agents on our findings. As no studies have documented reliable values of baPWV and $\mathrm{rbPEP} / \mathrm{rbET}$ indicating $\mathrm{LV}$ diastolic and systolic dysfunction, we used median values of baPWV and rbPEP/ rbET to classify our study patients. In addition, we classified the subjects on the basis of non-invasive data rather than by invasive measurements. The results may be somewhat different if invasive data are used for group classification.

\section{Conclusions}

The present study demonstrated that $\mathrm{rbPEP} / \mathrm{rbET}$, a surrogate of LV systolic function, had an important impact on the relationship between baPWV and LV diastolic function. In patients with high rbPEP/rbET but low baPWV, low baPWV may not indicate good LV diastolic function but implies that cardiac dysfunction may precede vascular dysfunction in such patients. When interpreting the relationship between baPWV and LV diastolic function, the rbPEP/rbET obtained from the same examination should be considered.

\section{CONFLICT OF INTEREST}

The authors declare no conflict of interest.

\section{ACKNOWLEDGEMENTS}

The research presented in this article was supported by a grant from Kaohsiung Municipal Hsiao-Kang Hospital (kmhk-97-018), Kaohsiung Medical University, Kaohsiung, Taiwan.

1 Mattace-Raso FU, van der Cammen TJ, Hofman A, van Popele NM, Bos ML, Schalekamp MA, Asmar R, Reneman RS, Hoeks AP, Breteler MM, Witteman JC. Arterial stiffness and risk of coronary heart disease and stroke: the Rotterdam Study. Circulation 2006; 113: 657-663.
2 Willum-Hansen T, Staessen JA, Torp-Pedersen C, Rasmussen S, Thijs L, Ibsen H, Jeppesen J. Prognostic value of aortic pulse wave velocity as index of arterial stiffness in the general population. Circulation 2006; 113: 664-670.

3 Imanishi R, Seto S, Toda G, Yoshida M, Ohtsuru A, Koide Y, Baba T, Yano K. High brachial-ankle pulse wave velocity is an independent predictor of the presence of coronary artery disease in men. Hypertens Res 2004; 27: 71-78.

4 Masugata H, Senda S, Yoshikawa K, Yoshihara Y, Daikuhara H, Ayada Y, Matsushita H, Nakamura H, Taoka T, Kohno M. Relationships between echocardiographic findings, pulse wave velocity, and carotid atherosclerosis in type 2 diabetic patients. Hypertens Res 2005; 28: 965-971.

5 Abhayaratna WP, Srikusalanukul W, Budge MM. Aortic stiffness for the detection of preclinical left ventricular diastolic dysfunction: pulse wave velocity versus pulse pressure. J Hypertens 2008; 26: 758-764.

6 Eren M, Gorgulu S, Uslu N, Celik S, Dagdeviren B, Tezel T. Relation between aortic stiffness and left ventricular diastolic function in patients with hypertension, diabetes, or both. Heart 2004; 90: 37-43.

7 Abhayaratna WP, Barnes ME, O'Rourke MF, Gersh BJ, Seward JB, Miyasaka Y, Bailey $K R$, Tsang TS. Relation of arterial stiffness to left ventricular diastolic function and cardiovascular risk prediction in patients $>$ or $=65$ years of age. Am J Cardiol 2006; 98: 1387-1392.

8 Yambe M, Tomiyama H, Hirayama Y, Gulniza Z, Takata Y, Koji Y, Motobe K, Yamashina A. Arterial stiffening as a possible risk factor for both atherosclerosis and diastolic heart failure. Hypertens Res 2004; 27: 625-631.

9 Hsu PC, Lin TH, Lee CS, Lee HC, Chu CY, Su HM, Voon WC, Lai WT, Sheu SH. Mismatch between arterial stiffness increase and left ventricular diastolic dysfunction. Heart Vessels 2010; 25: 485-492.

10 Weber T, Auer J, Lamm G, O'Rourke MF, Eber B. Arterial stiffness, central blood pressures, and wave reflections in cardiomyopathy-implications for risk stratification. $J$ Card Fail 2007; 13: 353-359.

11 Yamashina A, Tomiyama H, Takeda K, Tsuda H, Arai T, Hirose K, Koji Y, Hori S, Yamamoto $Y$. Validity, reproducibility, and clinical significance of noninvasive brachialankle pulse wave velocity measurement. Hypertens Res 2002; 25: 359-364.

12 Su HM, Lin TH, Lee CS, Lee HC, Chu CY, Hsu PC, Voon WC, Lai WT, Sheu SH. Myocardial performance index derived from brachial-ankle pulse wave velocity: a novel and feasible parameter in evaluation of cardiac performance. Am J Hypertens 2009; 22: 871-876.

13 Abhayaratna WP, Marwick TH, Smith WT, Becker NG. Characteristics of left ventricular diastolic dysfunction in the community: an echocardiographic survey. Heart 2006; 92: 1259-1264.

14 Khouri SJ, Maly GT, Suh DD, Walsh TE. A practical approach to the echocardiographic evaluation of diastolic function. J Am Soc Echocardiogr 2004; 17: 290-297.

15 Lang RM, Bierig M, Devereux RB, Flachskampf FA, Foster E, Pellikka PA, Picard MH, Roman MJ, Seward J, Shanewise JS, Solomon SD, Spencer KT, Sutton MS, Stewart WJ, Chamber Quantification Writing Group; American Society of Echocardiography's Guidelines and Standards Committee; European Association of Echocardiography. Recommendations for chamber quantification: a report from the American Society of Echocardiography's Guidelines and Standards Committee and the Chamber Quantification Writing Group, developed in conjunction with the European Association of Echocardiography, a branch of the European Society of Cardiology. J Am Soc Echocardiogr 2005; 18: 1440-1463.

16 Tomiyama H, Yamashina A, Arai T, Hirose K, Koji Y, Chikamori T, Hori S, Yamamoto Y, Doba N, Hinohara S. Influences of age and gender on results of noninvasive brachialankle pulse wave velocity measurement-a survey of 12517 subjects. Atherosclerosis 2003; 166: 303-309.

17 Yokoyama H, Shoji T, Kimoto E, Shinohara K, Tanaka S, Koyama H, Emoto M, Nishizawa Y. Pulse wave velocity in lower-limb arteries among diabetic patients with peripheral arterial disease. J Atheroscler Thromb 2003; 10: 253-258.

18 Chen SC, Chang JM, Tsai JC, Hsu PC, Lin TH, Su HM, Voon WC, Hwang SJ, Chen HC. A new systolic parameter defined as the ratio of brachial pre-ejection period to brachial ejection time predicts overall and cardiovascular mortality in hemodialysis patients. Hypertens Res 2010; 33: 492-498.

19 Garcia MJ, Thomas JD, Klein AL. New Doppler echocardiographic applications for the study of diastolic function. J Am Coll Cardiol 1998; 32: 865-875.

20 Nagueh SF, Middleton KJ, Kopelen HA, Zoghbi WA, Quiñones MA. Doppler tissue imaging: a noninvasive technique for evaluation of left ventricular relaxation and estimation of filling pressures. J Am Coll Cardiol 1997; 30: 1527-1533.

21 Tsang TS, Barnes ME, Gersh BJ, Bailey KR, Seward JB. Left atrial volume as a morphophysiologic expression of left ventricular diastolic dysfunction and relation to cardiovascular risk burden. Am J Cardiol 2002; 90: 1284-1289.

22 Pritchett AM, Mahoney DW, Jacobsen SJ, Rodeheffer RJ, Karon BL, Redfield MM. Diastolic dysfunction and left atrial volume: a population-based study. J Am Coll Cardiol 2005; 45: 87-92.

23 Ommen SR, Nishimura RA, Appleton CP, Miller FA, Oh JK, Redfield MM, Tajik AJ. Clinical utility of Doppler echocardiography and tissue Doppler imaging in the estimation of left ventricular filling pressures: a comparative simultaneous Doppler-catheterization study. Circulation 2002; 102: 1788-1794.

24 Su HM, Lin TH, Voon WC, Lee KT, Chu CS, Lai WT, Sheu SH. Differentiation of left ventricular diastolic dysfunction, identification of pseudonormal/restrictive mitral inflow pattern and determination of left ventricular filling pressure by Tei index obtained from tissue Doppler echocardiography. Echocardiography 2006; 23: 287-294.

25 Sutton-Tyrrell K, Mackey RH, Holubkov R, Vaitkevicius PV, Spurgeon HA, Lakatta EG. Measurement variation of aortic pulse wave velocity in the elderly. Am J Hypertens $2001 ; 14: 463-468$. 
26 Wilkinson IB, Fuchs SA, Jansen IM, Spratt JC, Murray GD, Cockcroft JR, Webb DJ. Reproducibility of pulse wave velocity and augmentation index measured by pulse wave analysis. J Hypertens 1998; 16: 2079-2084.

27 Asmar R, Benetos A, Topouchian J, Laurent P, Pannier B, Brisac AM, Target R, Levy BI. Assessment of arterial distensibility by automatic pulse wave velocity measurement: validation and clinical application studies. Hypertension 1995; 26: 485-490.

28 Mohiaddin RH, Firmin DN, Longmore DB. Age-related changes of human aortic flow wave velocity measured noninvasively by magnetic resonance imaging. $J$ Appl Physiol 1993; 74: 492-497.

29 Miyano I, Nishinaga M, Takata J, Shimizu Y, Okumiya K, Matsubayashi K, Ozawa T, Sugiura T, Yasuda N, Doi Y. Association between brachial-ankle pulse wave velocity and 3-year mortality in community-dwelling older adults. Hypertens Res 2010; 33: 678-682.

30 Tanaka H, Munakata M, Kawano Y, Ohishi M, Shoji T, Sugawara J, Tomiyama H, Yamashina A, Yasuda H, Sawayama T, Ozawa T. Comparison between carotid-femoral and brachial-ankle pulse wave velocity as measures of arterial stiffness. $J$ Hypertens 2009; 27: 2022-2027.
31 McEniery CM, Yasmin, Hall IR, Qasem A, Wilkinson IB, Cockcroft JR, ACCT Investigators. Normal vascular aging: differential effects on wave reflection and aortic pulse wave velocity: the Anglo-Cardiff Collaborative Trial (ACCT). J Am Coll Cardiol 2005; 46: 1753-1760.

32 Munagala VK, Jacobsen SJ, Mahoney DW, Rodeheffer RJ, Bailey KR, Redfield MM. Association of newer diastolic function parameters with age in healthy subjects: a population-based study. J Am Soc Echocardiogr 2003; 16: 1049-1056.

33 McEniery CM, Yasmin, Hall IR, Qasem A, Wilkinson IB, Cockcroft JR, ACCT Investigators. Normal vascular aging: differential effects on wave reflection and aortic pulse wave velocity: the Anglo-Cardiff Collaborative Trial (ACCT). J Am Coll Cardiol 2005; 46: 1753-1760.

34 Munagala VK, Jacobsen SJ, Mahoney DW, Rodeheffer RJ, Bailey KR, Redfield MM. Association of newer diastolic function parameters with age in healthy subjects: a population-based study. J Am Soc Echocardiogr 2003; 16: 1049-1056.

35 Yamada H, Oki T, Mishiro Y, Tabata T, Abe M, Onose Y, Wakatsuki T, Ito S. Effect of aging on diastolic left ventricular myocardial velocities measured by pulsed tissue Doppler imaging in healthy subjects. J Am Soc Echocardiogr 1999; 12: 574-581. 